\title{
The Uptake Mechanism of the Cell-Penetrating pVEC Peptide
}

\author{
Ihsan Omur Akdag and Elif Ozkirimli \\ Chemical Engineering Department, Bogazici University, Bebek, 34342 Istanbul, Turkey \\ Correspondence should be addressed to Elif Ozkirimli; elif.ozkirimli@boun.edu.tr
}

Received 17 December 2012; Revised 8 March 2013; Accepted 10 March 2013

Academic Editor: Yifat Miller

Copyright (C) 2013 I. O. Akdag and E. Ozkirimli. This is an open access article distributed under the Creative Commons Attribution License, which permits unrestricted use, distribution, and reproduction in any medium, provided the original work is properly cited.

\begin{abstract}
Peptide based drug design efforts have gained renewed interest with the discovery of cargo-carrying or cell-penetrating peptides. Understanding the translocation mechanism of these peptides and identifying the residues or elements that contribute to uptake can provide valuable clues toward the design of novel peptides. To this end, we have performed steered molecular dynamics (SMD) simulations on the pVEC peptide from murine vascular endothelial-cadherin protein and its two variants. Translocation was found to occur in three stages, adsorption via the cationic residues, inclusion of the whole peptide inside the membrane accompanied by formation of a water defect, and exit of both peptide and water molecules from the bilayer. Our simulation results suggest that the precise order in which the hydrophobic, cationic, and the polar regions are located in the amphipathic pVEC peptide contributes to its uptake mechanism. These results present new opportunities for the design of novel cell-penetrating and antimicrobial peptides.
\end{abstract}

\section{Introduction}

An ideal drug is only effective insofar as its ability to reach its target. Peptide drugs, with their high specificity and affinity to their targets, have emerged as promising drug leads $[1,2]$. However, most peptides, as well as proteins and oligonucleotides, are mainly hydrophilic, hindering their cellular uptake and, hence, limiting their widespread use as therapeutics [3, 4]. Delivery of these compounds into the cell is an intense area of research, and microinjection [5], electroporation [6], liposomes [7], endocytosis [8], and viral vectors have been previously proposed as potential delivery methods [9]. However, these delivery methods are not efficient, and accordingly most of the peptides in the market target extracellular enzymes or receptors. The discovery of cargo-carrying or cell-penetrating peptides (CPPs) $[10,11]$ has spurred renewed interest in the use of peptide drugs against intracellular targets. These peptides are able to penetrate the cell membrane and carry cargo of different size and hydrophobicity into the cell. CPPs are cationic and amphipathic peptides of usually fewer than 30 amino acids $[12,13]$. They do not share an obvious common sequence or structural motif [12]. Although the translocation mechanism is not the same for different CPP families, there are two major cellular uptake mechanisms, which are direct penetration (energy-independent) and endocytotic pathways [14]. In an energy- and receptor-independent mechanism, they penetrate cell membranes either via electrostatic interactions or hydrogen bonding $[9,15-17]$. The spontaneous penetration of CPPs may be by the carpet model, through transient pores, through the formation of inverted micelles, local electroporation, and direct insertion of the unfolded peptide into the membrane $[10,12,13]$. The uptake mechanism at an atomic level of detail is not known, and some questions of significance for peptide design remain. Which residues or structural elements contribute to uptake? Which interactions allow and/or facilitate transport of the peptide? Here, we examine the membrane translocation mechanism of one such peptide, pVEC, using steered molecular dynamics (SMD) simulations.

pVEC, an 18-amino-acid-long CPP, is derived from murine vascular endothelial-cadherin protein that functions in the physical contact between adjacent cells. With the sequence, LLIILRRRIRKQAHAHSK, the N-terminus is hydrophobic, the middle part is charged, and the C-terminus is hydrophilic, making it an amphipathic molecule. Previous cargo-carrying experiments showed that pVEC was able to carry some proteins and oligomers [18]. pVEC was found to have a random coil structure in pure water and neutral phospholipids, but it forms a beta-sheet in the negatively 
charged DOPG membrane [16]. Structure-activity relationship (SAR) experiments, in which each pVEC residue was mutated to L-alanine and the change in the cellular uptake of pVEC into human Bowes melanoma cells was examined, were performed in order to identify the contribution of each residue to uptake [18]. Substitution of the five Nterminal residues to L-alanine was shown to drop cellular uptake by $50 \%$ to $75 \%$ suggesting that the hydrophobic Nterminus of the peptide is important for cellular uptake. Furthermore, deletion of the three N-terminal hydrophobic residues completely abolished uptake, and when all five hydrophobic residues (LLIIL) were replaced by L-alanines in the N-terminus, the uptake of peptide was not detected at all.

The behavior of peptides in or near lipid bilayer environments has been previously investigated using molecular dynamics (MD) simulations. However, MD simulations are not sufficiently long to sample events, such as transport across the membrane, that take place on the microsecond or longer timescale, and nonequilibrium MD simulations provide an attractive alternative to obtain atomic level detail about such transitions. One of these nonequilibrium methods is steered molecular dynamics (SMD) [19-21], in which force is applied to one (or more) SMD atom to move the system from one state to another. This nonequilibrium method has been used with success in a variety of dynamic systems to study drug binding [22], transport across membrane proteins [23], and unfolding [24]. SMD simulations have also been used to examine peptide uptake through the lipid bilayer, as $\mathrm{MD}$ simulations on the cardiotoxin A3 peptide and POPC lipid bilayer system showed that the peptide would not spontaneously bind to the membrane [25]. SMD simulations showed that Tryptophan residue of the WL5, with the sequence WLLLLL, was found to contribute to peptide-membrane interactions, and penetration depth of the peptide into the membrane was found to be related to its hydrophobicity [26]. Similarly, the presence of hydrophobic tryptophan residues was found to result in deeper insertion of Indolicidin, an antimicrobial peptide, while positive charges were critical to the peptide's adsorption [27].

Here, SMD simulations on wild type pVEC and its two variants were performed to move the peptide from one side of the bilayer to the other. The translocation mechanism was observed to occur in three distinct stages; the insertion of the N-terminus into the bilayer, the inclusion of the whole peptide inside the membrane, and the exit of the peptide from the bilayer. The presence of barriers at these stages was verified by the presence of plateaus followed by abrupt changes in the interaction energy profiles as well as sudden increases in force applied to the SMD atom. The structural elements that contribute to peptide-membrane association and subsequent uptake were identified.

\section{Methods}

2.1. Simulation Systems. The initial coordinates of the pVEC peptide were assigned as a beta-hairpin starting from the coordinates of a beta-hairpin loop of a beta-lactamase inhibitor protein using the psfgen module of visual molecular
TABLE 1: The pVEC sequences used in the simulations and the mutated residue for each sequence.

\begin{tabular}{lc}
\hline Name & \multicolumn{1}{c}{ Sequence } \\
\hline pVEC & LLIILRRRIRKQAHAHSK \\
Retro-pVEC & KSHAHAQKRIRRRLIILL \\
Scramble-pVEC & IAARIKLRSRQHIKLRHL \\
\hline
\end{tabular}

dynamics (VMD) [28]. Using the modeled pVEC coordinates as a template, the sequence was altered to obtain the retropVEC and scramble-pVEC coordinates. The peptide pVEC (wild-type), retro-pVEC, which has the same sequence in reverse order, and scramble-pVEC, which has the same amino acid content in random order [29], were studied (Table 1).

2.2. Equilibration of Peptide in Water Environment. The pVEC peptide (or its variants) was solvated with the solvate module in VMD using $10 \AA$ padding in each direction resulting in a water box size of $43 \AA \times 48 \AA \times 52 \AA$. Water molecules closer than $2.8 \AA$ to the peptide were removed. The waterpeptide system was minimized for 1000 steps and equilibrated for $2 \mathrm{~ns}$ with harmonic constraints $\left(k=1 \mathrm{kcal} / \mathrm{mol} / \AA^{2}\right)$ on peptide atoms. Then, all atoms of the system were allowed to equilibrate for $2 \mathrm{~ns}$ without constraints.

2.3. Preparation of Lipid-Water-Peptide System. The membrane was created in $50 \AA$ in $x$ - and $50 \AA$ in $y$-directions. The membrane lipid was chosen to be POPE in order to mimic the membrane of E. coli [30]. The membrane was solvated with TIP3P water molecules $80 \AA$ in positive and negative $z$-directions. After solvation, the water-membrane system was equilibrated for $0.5 \mathrm{~ns}$ with harmonic constraints $\left(k=1 \mathrm{kcal} / \mathrm{mol} / \AA^{2}\right)$ and for $0.5 \mathrm{~ns}$ without constrains. The previously equilibrated peptide was placed into the water layer above the membrane manually such that the minimum distance between the peptide atoms and the membrane phosphate atoms was more than $10 \AA$. Water molecules closer than $2.8 \AA$ to the peptide were removed. Counterions were added to a concentration of $0.4 \mathrm{M}$.

2.4. Steered Molecular Dynamics Simulations. Steered molecular dynamics simulations [22] were performed by applying force on the $\mathrm{N}$-terminal $\mathrm{C}_{\alpha}$ atom (SMD atom) of the peptide in the $z$-direction to move the peptide across the membrane. For these simulations, the phosphorous heads of the lipid bilayer were harmonically constrained in the $z$-direction with a force constant of $1 \mathrm{kcal} / \mathrm{mol} / \AA^{2}$, while they were free to move in $x$ - and $y$-directions allowing the formation of the pore through which the peptide and/or water entered the bilayer. The spring constant in the SMD simulations was $10 \mathrm{kcal} / \mathrm{mol} / \AA^{2}$, and the velocity of pulling was $0.0000050 \AA /$ timestep which corresponds to $2.50 \AA / \mathrm{ns}$ with timestep $=2.0 \mathrm{fs}$. The peptide traveled $120 \AA$ to completely traverse the membrane within about $50 \mathrm{~ns}$ simulation time. 

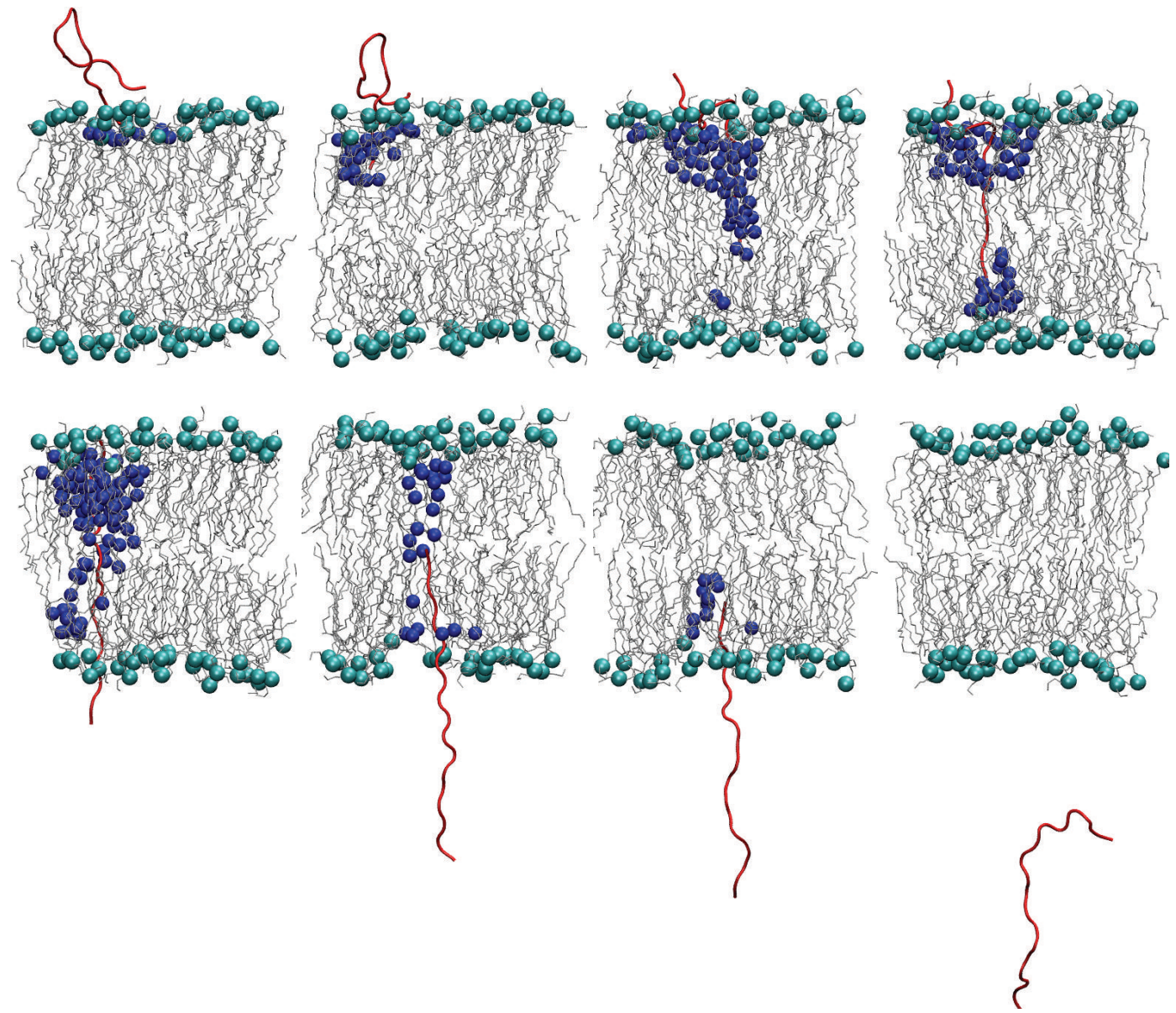

FIGURE 1: Snapshots for the SMD simulation on pVEC peptide as it moves across the bilayer. The peptide backbone is shown in red in cartoon representation, the phosphate heads are shown as cyan spheres, the lipid tails are shown in gray, and the water molecules are shown as blue spheres. Hydrogens in the lipids and water molecules are omitted for clarity.

TABLE 2: Summary of events in the SMD simulation on pVEC with SMD force applied on the N-terminal $\mathrm{C}_{\alpha}$ atom.

\begin{tabular}{|c|c|c|c|c|c|}
\hline & $z(\AA)$ & Peptide location & $\begin{array}{l}\text { Interaction energy } \\
(\mathrm{kcal} / \mathrm{mol})\end{array}$ & Force $(\mathrm{pN})$ & $\begin{array}{l}\text { Number of waters } \\
\text { in membrane }\end{array}$ \\
\hline (i) & 30 & Before entry & 0 & 0 & 0 \\
\hline (ii) & 20 & First contact with membrane & -300 & -1000 & 0 \\
\hline (iii) & 10 & SMD atom through the $\mathrm{P}$ layer & -100 & -1000 & 10 \\
\hline (iv) & 0 to -20 & $\begin{array}{l}\text { Peptide moves through membrane, } \\
\text { N-terminus reaches lower P layer }\end{array}$ & -500 & -1000 & 20 to 5 \\
\hline (v) & -30 to -60 & Peptide continues to move out & -300 & -500 & 60 to 10 \\
\hline
\end{tabular}

All simulations were calculated with the NAMD program [31] using the CHARMM27 potential energy function for the all atom model for proteins [32] and lipids [33] and TIP3P water parameters [34]. Periodic boundary conditions with particle mesh Ewald [35] method were used. Nonbonded interactions were truncated at a cutoff value of $12 \AA$ and a switching distance of $10 \AA$. Nonbonded interactions were calculated at each step. SHAKE was applied on all hydrogen distances and a timestep of $2 \mathrm{fs}$ was used. Constant pressure of $1 \mathrm{~atm}$ was imposed by the Langevin piston $[36,37]$ with a piston period of $200 \mathrm{fs}$. Langevin dynamics were used to keep the system at constant temperature $300 \mathrm{~K}$ with a damping coefficient of $5 / \mathrm{ps}$. The coordinate sets were saved every $2 \mathrm{ps}$ for analysis. The SMD forces were output every 20 steps (40 fs).

2.5. Trajectory Analysis. Interaction energy between the peptide and the lipids was calculated using the NAMD Energy plugin of VMD. Force was calculated by NAMD at every SMD step. Work was calculated as the integral of force multiplied by pulling velocity over time. The number of water molecules within the membrane boundaries was monitored. Secondary 


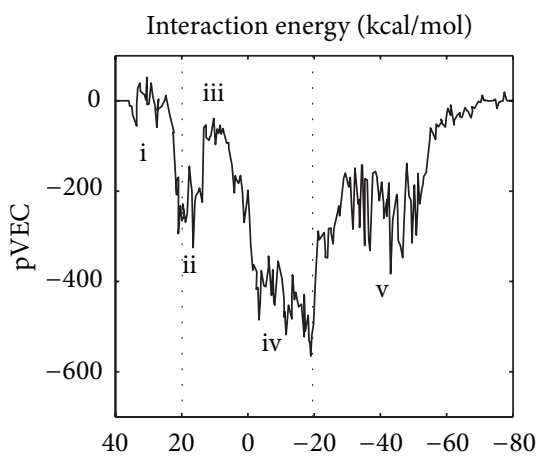

(a)

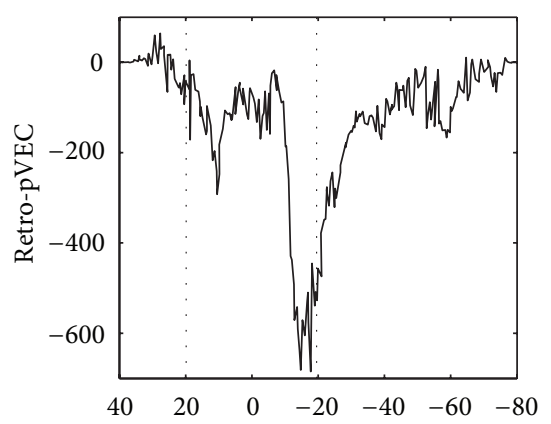

(d)

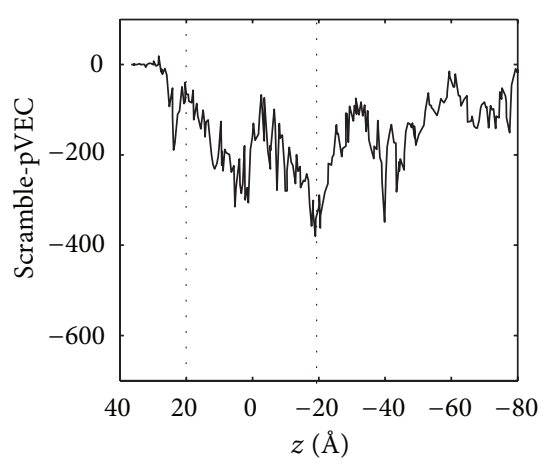

(g)

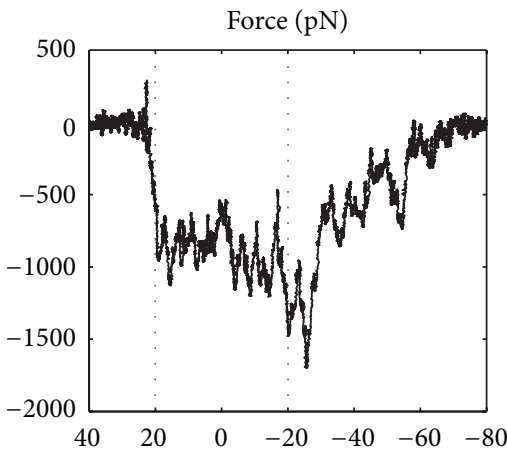

(b)

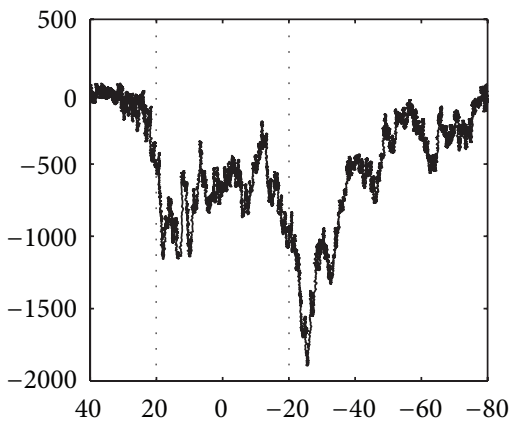

(e)

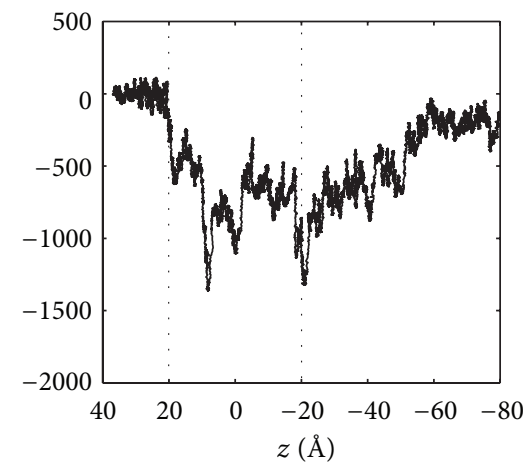

(h)

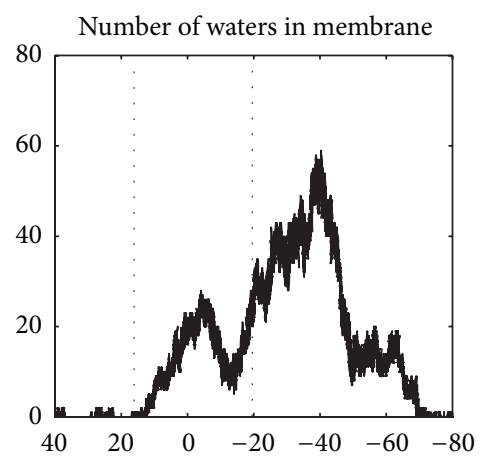

(c)

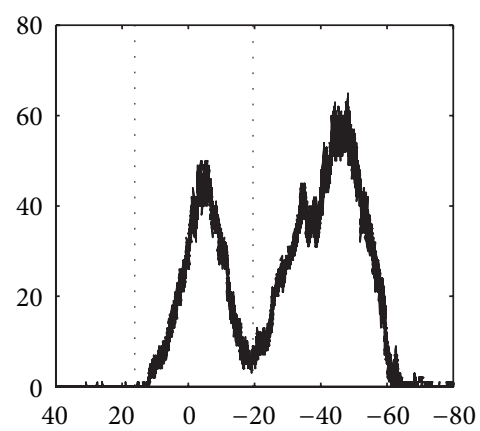

(f)

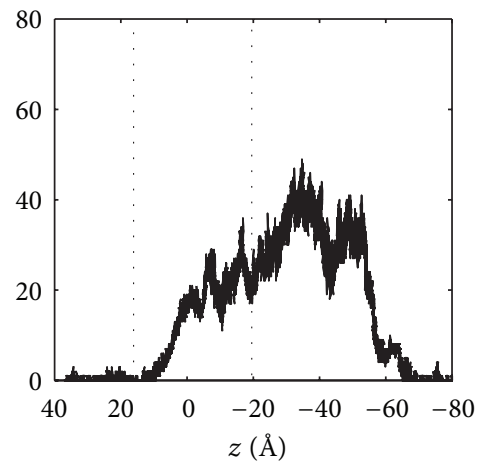

(i)

FIGURE 2: Interaction energy between the lipid and peptide atoms (left panel), force applied to the SMD atom (middle panel), and the number of water molecules in the membrane boundary (right panel) is shown as a function of the SMD atom $z$-coordinate for SMD simulations on the pVEC (top), retro-pVEC (middle), and scramble-pVEC (bottom) transport across the lipid bilayer. The membrane boundaries are indicated by the dashed lines at $z=20 \AA$ and $-20 \AA$. The regions in which the interaction energy between wild type pVEC and the membrane stays constant for more than $5 \mathrm{~ns}$ are indicated in panel (a).

structure of the peptide during the SMD simulations was monitored using the Timeline tool of VMD. Structure figures were prepared with VMD [28].

\section{Results and Discussion}

Steered molecular dynamics simulations on the wild-type pVEC, retro-pVEC, which has the pVEC sequence in reverse order, and scramble-pVEC, which has the same amino acid composition but a scrambled sequence (Table 1), were performed and analyzed to obtain atomic level information on the translocation mechanism of pVEC. The retro-pVEC and scramble-pVEC were shown to have dramatically reduced uptake compared to pVEC as measured by the level of fluorescence in the cells after incubation with fluoresceinated peptide [29], and we aimed to elucidate the reasons for the drop in uptake values observed for these peptides using SMD simulations and comparing the results with those of pVEC simulations.

As a first step, SMD trajectories were analyzed visually to obtain information about the overall structural changes in peptide, membrane, or water. Some snapshots from the 
TABLE 3: The maximum interaction energy, maximum force applied, and maximum work done values for the pVEC simulations.

\begin{tabular}{lccc}
\hline Name & $\begin{array}{c}\text { Maximum } \\
\text { interaction } \\
\text { energy }(\mathrm{kcal} / \mathrm{mol})\end{array}$ & $\begin{array}{c}\text { Maximum force } \\
\text { applied in } \\
\text { negative } z \\
\text { direction }(\mathrm{pN})\end{array}$ & $\begin{array}{c}\text { Maximum } \\
\text { work done } \\
(\mathrm{kcal} / \mathrm{mol})\end{array}$ \\
\hline pVEC & -565 & 1653 & 916 \\
$\begin{array}{l}\text { Retro-pVEC } \\
\text { Scramble- }\end{array}$ & -685 & 1803 & 919 \\
pVEC & -380 & 1308 & 797 \\
\hline
\end{tabular}

trajectory are shown in Figure 1. The first noteworthy change was in the peptide conformation. pVEC initial structure was assigned as a beta-hairpin. pVEC was reported to have a random coil structure in pure water and neutral phospholipids, and to form a beta-sheet in negatively charged DOPG membrane [16]. In the simulations, the SMD force was applied to the $\mathrm{N}$-terminal $\mathrm{C}_{\alpha}$ atom to facilitate transport of the peptide in $50 \mathrm{~ns}$, while the rest of the peptide was allowed to move freely and therefore could maintain its betasheet form. Examination of the change in secondary structure during the simulations (Supplementary Figure 2) showed that the peptide remained in the beta-sheet form until the SMD atom entered the bilayer, after which it gradually unfolded into a random coil and moved across the bilayer as a chain. Upon exit, the peptide started to become more compact, as shown by the decrease in the distance between the $\mathrm{N}$ - and Cterminal $\mathrm{C}_{\alpha}$ atoms (Supplementary Figure 2), even though the only force continued to be on the SMD atom. Our results suggest that the unfolding of the peptide, which is observed during peptide transport across the bilayer, is due to the interactions with membrane atoms, which were represented with an explicit model in this work. The second structural change was the formation of a water defect as the peptide travelled across the bilayer. Formation of the water defect may alter the membrane permeability, enhancing uptake. The mechanism by which the changes in the peptide and the membrane structure may contribute to uptake is examined in detail in the following sections.

3.1. pVEC Travels across the Lipid Bilayer in Three Stages. The uptake of the pVEC peptide across the POPE bilayer as the $\mathrm{C}_{\alpha}$ atom of the peptide $\mathrm{N}$-terminal residue was pulled across the bilayer was monitored. The interaction energy between the peptide, and the lipid bilayer, the force applied to the peptide and the number of water molecules within the lipid bilayer were calculated and plotted as a function of the $z$-coordinate of the SMD atom (Figure 2).

The interaction energy profile for the wild-type pVEC simulation contains regions in which the energy stays constant for more than $5 \mathrm{~ns}$ (indicated as $\mathrm{i}$ through $\mathrm{v}$ in Figure 2(a)) followed by sudden increases or decreases. The "plateaus" in the interaction energy profile (Figure 2(a) and ii, iv, and v) may indicate regions of sustained interaction that must break for the transport to proceed, or, namely, energy barriers in the process. Similarly, the abrupt increases in the force profile observed at entrance and exit of SMD atom into the bilayer (Figure 2(b); $z \approx 20$ and $-25 \AA$ ) may point out the possible energy barriers that must be overcome by the application of increased force. These changes in energy and force suggest that pVEC transport occurs in three main stages, which are the adsorption of the peptide at the lipidwater interface $(z \approx 20 \AA$, ii), the inclusion of the whole peptide inside the membrane $(z \approx 0$ to $-20 \AA$, iv), and the exit of the peptide $\mathrm{N}$-terminus from the bilayer followed by exit of the whole peptide ( $z \approx-30$ to $-60 \AA$, v) with the main barriers to transport at the membrane-water boundaries on both sides. The steps are summarized in Table 2 and explained in some detail later.

Before contact with the bilayer $(z \approx 30 \AA$; Figure 2(a), $i)$, the interaction energy, force, and number of waters in the membrane are all zero. When the peptide makes its first contact with the membrane, the interaction energy reaches its first maximum value at about $-300 \mathrm{kcal} / \mathrm{mol}$ as a result of the interaction of the positively charged residues and the positively charged amino terminus with the phosphate groups and the first barrier is observed (Figure 2(a) and ii). Even though the SMD force is applied to the N-terminal $\mathrm{C}_{\alpha}$ atom, the positively charged residues of the peptide midsection also make contact with the negatively charged phosphate layer. Once the $\mathrm{N}$-terminus passes the $\mathrm{P}$ heads of the membrane ( $z \approx 10 \AA$; Figure $2(\mathrm{a})$ and iii), the interaction energy decreases back to about $-100 \mathrm{kcal} / \mathrm{mol}$ and peptide insertion starts. Between $z=0$ and $-20 \AA$, the peptide moves through the bilayer (Figure 2(a) and iv). The second barrier is seen at this stage and the interaction energy reaches its overall maximum point at around $-500 \mathrm{kcal} / \mathrm{mol}$. At $z \approx$ $-20 \AA$, the $\mathrm{N}$-terminus is in contact with the lower P heads while the $\mathrm{C}$-terminus is in contact with the upper $\mathrm{P}$ heads. As the peptide moves out of the lipid bilayer $(z \approx-30$ to $-60 \AA$; Figure 2(a) and v), interaction between the peptide and the membrane decreases back to about $-300 \mathrm{kcal} / \mathrm{mol}$ and oscillates around this value as the peptide tries to find its way out of the membrane. The interaction between the peptide and the membrane is maintained until the peptide completely leaves the membrane at $z \approx-70 \AA$ and starts to become more compact (Supplementary Figure 2).

The electrostatic and van der Waals energies between the peptide and the membrane were also calculated to identify the contribution of each to the total interaction energy (Supplementary Figure 1). The electrostatic energy contribution to the interaction energy profile was found to be higher and the electrostatic energy profile resembles that of the total interaction energy, with maximum energy value attained when the peptide is completely immersed inside the membrane. At this point, the van der Waals energy also contributes $100 \mathrm{kcal} / \mathrm{mol}$ to total energy.

The SMD force applied to the wild-type pVEC in the negative $z$-direction during the simulations was calculated and plotted with respect to SMD atom $z$-coordinate (Figure 2(b)). At the beginning of the simulation, as the peptide moves through the water layer, the magnitude of the force applied is $0 \mathrm{pN}$. At $z \approx 20 \AA$, a sudden increase in the force is observed as the peptide contacts the membrane. The membrane resists penetration by the peptide, and to overcome this resistance, about $1000 \mathrm{pN}$ of force is applied to the peptide as it moves 
through the bilayer. The force value increases to $-1500 \mathrm{pN}$ $(z \approx-25 \AA)$ when the peptide is totally immersed in the membrane and the $\mathrm{N}$-terminus starts to exit the membrane. After this checkpoint, the peptide starts to leave the membrane and a lower force of about $-500 \mathrm{pN}$ is applied until the peptide is out of the membrane and moving through the water layer, at which point the magnitude of the force applied reduces back to $0 \mathrm{pN}$.

The number of water molecules that penetrate into the cell membrane was monitored (Figure 2(c)). At the beginning of the simulation, the peptide is embedded in the water layer above the membrane and the initial number of water molecules in the membrane is equal to zero until the peptide starts to penetrate into the membrane $(z \approx 20 \AA)$. As peptide insertion starts, the cavity formed by the peptide is filled by about 20 water molecules causing the small peak observed at $z \approx 0 \AA$. As peptide insertion continues and the SMD atom approaches the lower layer of the membrane, some of the water molecules grouped around the first residues of the peptide leave the membrane and the number of water molecules drop $(z \approx-13 \AA)$. When the SMD atom starts to leave the membrane, the C-terminal of the peptide is in contact with the upper layer of the membrane and the peptide is completely extended. As the peptide beta-hairpin structure unfolds and the peptide is immersed in the membrane, the number of water molecules in the membrane increases and reaches its maximum at around 70 at $z \approx-40 \AA$. The formation of the larger water defect is due to solvation of the cationic stretch (RRRIRK) in the midsection of pVEC. After this peak, a small shoulder is observed between $z=-50$ and $-70 \AA$, and about 20 waters are retained in the membrane as the peptide continues its exit. The number of water molecules continues to decrease and finally becomes 0 when the last residue Lys18 leaves the membrane.

There is some debate in the literature about whether arginine residues can be present in their ionized states in hydrophobic environments such as membrane proteins [38], peptides [39], or even protein interiors [40]. It has recently been shown by Tieleman and coworkers, who used molecular dynamics simulations to decipher the partitioning of amino acids between the water and lipid phase, that Arg can remain ionized even in the middle of the membrane due to formation of a water defect around the residue [41]. Furthermore, it has been suggested that additional arginines may take advantage of the existing water defect in a "piggyback" mechanism $[42,43]$. In the pVEC peptide, the cationic stretch of residues comprises a series of arginine residues. Mutation of these arginines to alanine (one at a time) has been shown to cause no drop in pVEC uptake potential [29]. However, the presence of the other arginines might be sufficient to form the initial contact, hence, promoting adsorption at the waterlipid interface, and then formation of the water defect, which may facilitate pore formation and transport.

\subsection{The Peptide Sequence Governs the Uptake Mechanism.} In an effort to understand how the pVEC peptide sequence, specifically the N-terminal hydrophobic stretch and the positively charged region in the middle, contributes to the uptake mechanism, the SMD simulations were repeated using retro-pVEC and scramble-pVEC. These two pVEC variants were shown to have lower uptake values as measured by accumulation of fluorescence [29]. The interaction energy, force, and number of water molecules in the membrane are shown in Figure 2 middle panel for retro-pVEC, and in Figure 2 bottom panel for scramble-pVEC and the maximum interaction energy values, the maximum force applied to the peptides, and the work values attained in the simulations are listed in Table 3 for each simulation.

The simulations on retro-pVEC were performed in order to compare how reversing the order in which residues enter the membrane would affect uptake. In the SMD simulation on pVEC, the SMD force is applied to the $\mathrm{N}$-terminal $\mathrm{C}_{\alpha}$ atom, and therefore the hydrophobic stretch enters first, then the cationic stretch, and lastly the polar stretch of residues travel through the membrane. In retro-pVEC, this order is opposite because even though the SMD force is applied on the $\mathrm{N}$-terminal $\mathrm{C}_{\alpha}$ atom again, the peptide sequence is in reverse order. It is not known which end moves across the bilayer first and comparison of the pVEC and retro-pVEC simulation results provide information about the consistently observed energy barriers for the two reaction coordinates. To further analyze entry at the polar C-terminus, the simulations on wild type pVEC were repeated by applying force on the C-terminal $\mathrm{C}_{\alpha}$ atom. The interaction energy, force, and number of waters were plotted as a function of the $z$-coordinate of the SMD atom in Supplementary Figure 3. Visual comparison of the trajectory as well as a comparison of the profiles for energy, force, and water defect size with the pVEC and retro-pVEC simulations showed that the three stages of the transition are also present in this simulation. A detailed comparison between the pVEC and retro-pVEC simulations, in both of which the SMD atom was the $\mathrm{N}$-terminal $\mathrm{C}_{\alpha}$ atom, is given later.

In the retro-pVEC simulations, the interaction energy has one pronounced peak at $z \approx-10 \AA$ and $-650 \mathrm{kcal} / \mathrm{mol}$ and two small plateaus at $z \approx 10 \AA$ and $-50 \AA$ and (Figure 2(d)). The initial drop in energy as well as two peaks (ii and v) observed in the pVEC simulations are missing in the retropVEC interaction energy profiles. The force profile also has a peak at $z \approx-10 \AA$ and $1750 \mathrm{pN}$ and two small plateaus at $z=20$ to $-20 \AA$ and -40 to $-60 \AA$, consistent with the interaction energy profile (Figure 2(e)). The maximum interaction energy and the maximum force applied are higher in retro-pVEC than in pVEC simulations (Table 3).

When the number of water molecules in the membrane is monitored during peptide transport in the pVEC simulation, three maxima $(z \approx 0,-40$, and $-60 \AA)$ are observed (Figure 2(c)). On the other hand, in the retro-pVEC simulation, the first maximum $(z \approx 0 \AA)$ is much higher at 50 water molecules (Figure 2(f)). The number of water molecules decreases at $z \approx-20 \AA$ and then increases again to 60 at $z \approx-40 \AA$. The initial abrupt increase observed in retro-pVEC is due to the uptake of water with the polar patch of residues that enters the membrane first, while in pVEC only the $\mathrm{N}$-terminus is positively charged, resulting in the low maximum followed by the high peak due to entrance of the posively charged midsection. 
It should be noted that three plateaus (albeit with different heights) are observed in the interaction energy profiles of pVEC and retro-pVEC simulations. Furthermore, the work values at the end of the simulations are similar at around $920 \mathrm{kcal} / \mathrm{mol}$. The similarity in overall properties may be due to the presence of the same the structural elements in both peptides, albeit in reverse order. The details of the interaction energy and force profiles as well as the formation of the water defect are different between pVEC and retropVEC simulations. In the pVEC simulation, the hydrophobic stretch is pulled through the membrane first, while in retropVEC simulations the polar patch is pulled through first. It has previously been suggested that hydrophobic groups contribute to deeper insertion of the peptide into the membrane [44]. Ile and Leu were shown by MacCallum et al. to be the most hydrophobic residues, preferring to be in the membrane bilayer rather than the interface [42]. Mutation of the LLIIL residues in pVEC one by one to alanine results in a decrease in uptake potential, while deletion of the initial three residues or replacing the first five residues by $\mathrm{L}$-alanine abolishes uptake [29]. These results suggest that the presence of the hydrophobic stretch composed of three Leu and two Ile residues, which are known to be highly hydrophobic, can contribute to penetration of pVEC into the bilayer. In our simulation results, when the hydrophobic stretch enters first in pVEC simulations, the interaction energy between the peptide and the membrane (Figure 2(a)) is lower than when the polar region enters first (Figure 2(d) and Supplementary Figure 3, left panel). The higher interaction energy between the polar stretch of residues and the membrane lower boundary may constitute a barrier to transition. Previous reports suggest that the hydrophobic stretch contributes to deeper penetration into the bilayer and facilitates transport. Furthermore, a large water defect that surrounds the $\mathrm{C}$-terminal polar patch needs to form in the retro-pVEC simulations (and in C-terminus first pVEC simulations, Supplementary Figure 3), while the water defect is smaller and grows in size as the peptide enters and the arginine rich cationic midsection moves through the bilayer. Therefore, peptide entry at its hydrophobic Nterminus, which "guides" the peptide into the hydrophobic membrane, may be a more facile process.

pVEC has three distinct regions that can be classified as hydrophobic, cationic, and polar, while scramble-pVEC has a completely random order, possibly altering the amphipathic character of the peptide. As a result, the energy, force, and water defect size profiles lack distinguishable regions observed in the previous simulations. The interaction energy profile has several distributed maxima (Figure 2(g)), mirrored by the force profile (Figure $2(\mathrm{~h})$ ). The two maxima observed at $z \approx 10 \AA$ and $-20 \AA$ in the force profile are due to interaction of the positively charged residues with the phosphate groups in the two leaflets. The maximum interaction energy value reached is lower than the two other simulations at $-380 \mathrm{kcal} / \mathrm{mol}$. The maximum force applied is also lower at $1300 \mathrm{pN}$. The flatter force profile as well as the low values of the maxima result in a low work value at $800 \mathrm{kcal} / \mathrm{mol}$.

In scramble-pVEC simulations, about 40 water molecules enter the bilayer with the peptide, the number of water molecules fluctuates between 20 and 40 with no pronounced peaks or troughs, and as the peptide leaves, the number drops gradually to 0 (Figure 2(i)). The absence of a well-defined structure in the number of water molecules that enter the membrane with the peptide is consistent with the relative absence of peaks in the interaction energy and force profiles.

\section{Conclusions}

In this study, steered molecular dynamics simulations on the pVEC peptide, retro-pVEC, and scramble-pVEC (Table 1) were performed in an effort to describe the mechanism whereby pVEC peptide is translocated across the cell membrane and to verify the significance of certain residues and structural elements in the translocation mechanism

Visual analysis of the trajectories showed that the peptide made its initial contact with the bilayer via its positively charged residues, and it unfolded from a beta-hairpin form to a random coil as it moved through the bilayer and that a water defect that surrounded the cationic or polar residues formed during peptide transport. The translocation mechanism was observed to occur in three main stages: (1) the insertion of the N-terminus into the bilayer, which is facilitated by the interaction of positively charged residues with the phosphate layer, (2) the inclusion of the peptide inside the membrane and formation of the water defect around the cationic stretch of residues, and (3) the exit of the peptide and water molecules from the bilayer.

The retro-pVEC uptake also has three stages represented as three maxima in the interaction energy profile, and similar amounts of work are applied in the pVEC and retro-pVEC simulations. The overall similarity in the two simulations correlate well with the presence of three distinct regions (hydrophobic-cationic-polar) in the two peptides. It is not known in which direction (N-terminus first or $\mathrm{C}$-terminus first) the peptide enters the lipid bilayer. However, the stronger interactions as well as the formation of a larger water defect observed in retro-pVEC simulations (and in SMD simulations with force applied on the C-terminus of pVEC) may suggest that polar C-terminal entry, rather than the hydrophobic N-terminal entry, is a difficult mechanism. The hydrophobic residues, which would prefer to be in the membrane as suggested by MacCallum and coworkers [42], may "pull" the rest of the peptide along the bilayer. It is important to note that nonequilibrium MD simulations sample a reaction coordinate above the free energy landscape, and therefore a comparison of the energy, force, or work numerical values, especially for single trajectories, may not provide accurate information about the free energy landscape of the transition [21, 45]. Multiple simulations are required to obtain more accurate estimates of free energy [46-48]. However, the consistently observed features in the transition pathways may be helpful in identifying energy barriers. Our major contribution is the observation that the presence of three distinct regions in pVEC or retro-pVEC is related to the three distinct stages of the transition and that the watermembrane boundaries are the main barriers to transition. 
On the other hand, the energy and force profiles of simulations on scramble-pVEC, which has no discernible sequence regions, do not have distinct peaks and are relatively more flat. The work that required to move the peptide across the membrane is also lower. This result would suggest relatively lower resistance to penetration; however, experiments have shown that this peptide has low uptake potential compared to pVEC [29]. It should be noted that, in $\mathrm{pVEC}$ and retro-pVEC simulations, an abrupt increase in force coincides with an increase in the interaction energy. Stronger interactions may create energy barriers that must be overcome, but the high interaction energy, especially between the cationic stretch and the top layer and later with the bottom layer, may also drive the peptide forward in the SMD direction. However, a continuously low force is applied in scramble-pVEC calculations, and no strong interactions form between the peptide and the membrane. Additionally, the water defect that forms is smaller in scramble-pVEC possibly due to the absence of an arginine rich cationic stretch of residues. There is no hydrophobic stretch of residues to move the peptide toward the membrane interior either. The absence of these two regions may cause the lower uptake observed for scramble-pVEC. We propose that it is either the residue composition nor the overall charge nor the overall hydrophobicity of the peptide but the presence of three distinct regions that contribute to the uptake of pVEC.

Our results provide evidence for unfolding of the peptide during direct penetration through bacterial membranes represented here by the explicit atoms of the POPE bilayer. The initial beta-hairpin form of the peptide is maintained in the water layer, but the peptide moves as a single chain during passage through the membrane. The membrane atoms act as a barrier in which the peptide and the surrounding water defect form a pore. Active participation of the membrane during uptake has been recognized [13], and our simulations also lend support to the use of explicit membrane models in the study of peptide transport.

\section{Abbreviations}

MD: $\quad$ Molecular dynamics

SMD: $\quad$ Steered molecular dynamics

POPE, CPP: Cell-penetrating peptide.

\section{Acknowledgments}

This work was supported by TUBITAK Research Grant 109M229 (E. Ozkirimli) and Bogazici University Research Grant 09HA504P (E. Ozkirimli). The authors thank Begum Alaybeyoglu for her help in figure preparation.

\section{References}

[1] P. Vanhee, A. M. van der Sloot, E. Verschueren, L. Serrano, F. Rousseau, and J. Schymkowitz, "Computational design of peptide ligands," Trends in Biotechnology, vol. 29, no. 5, pp. 231239, 2011.
[2] E. O. Olmez and B. S. Akbulut, "Protein-peptide interactions revolutionize drug development," in Binding Protein, K. Abdelmohsen, Ed., chapter 3, InTech, 2012.

[3] A. M. Gewirtz, D. L. Sokol, and M. Z. Ratajczak, "Nucleic acid therapeutics: state of the art and future prospects," Blood, vol. 92, no. 3, pp. 712-736, 1998.

[4] R. L. Juliano, S. Alahari, H. Yoo, R. Kole, and M. Cho, "Antisense pharmacodynamics: critical issues in the transport and delivery of antisense oligonucleotides," Pharmaceutical Research, vol. 16, no. 4, pp. 494-502, 1999.

[5] H. Arnheiter and O. Haller, "Antiviral state against influenza virus neutralized by microinjection of antibodies to interferoninduced Mx proteins," EMBO Journal, vol. 7, no. 5, pp. 1315-1320, 1988.

[6] R. Chakrabarti, D. E. Wylie, and S. M. Schuster, "Transfer of monoclonal antibodies into mammalian cells by electroporation," Journal of Biological Chemistry, vol. 264, no. 26, pp. 1549415500, 1989.

[7] R. M. Straubinger, N. Duzgunes, and D. Papahadjopoulos, "pHsensitive liposomes mediate cytoplasmic delivery of encapsulated macromolecules," FEBS Letters, vol. 179, no. 1, pp. 148-154, 1985.

[8] C. M. Varga, T. J. Wickham, and D. A. Lauffenburger, "Receptormediated targeting of gene delivery vectors: insights from molecular mechanisms for improved vehicle design," Biotechnology and Bioengineering, vol. 70, no. 6, pp. 593-605, 2000.

[9] B. Gupta, T. S. Levchenko, and V. P. Torchilin, "Intracellular delivery of large molecules and small particles by cellpenetrating proteins and peptides," Advanced Drug Delivery Reviews, vol. 57, no. 4, pp. 637-651, 2005.

[10] M. Magzoub and A. Gräslund, "Cell-penetrating peptides: small from inception to application," Quarterly Reviews of Biophysics, vol. 37, no. 2, pp. 147-195, 2004.

[11] F. Heitz, M. C. Morris, and G. Divita, “Twenty years of cellpenetrating peptides: from molecular mechanisms to therapeutics," British Journal of Pharmacology, vol. 157, no. 2, pp. 195-206, 2009.

[12] S. Yesylevskyy, S.-J. Marrink, and A. E. Mark, "Alternative mechanisms for the interaction of the cell-penetrating peptides penetratin and the TAT peptide with lipid bilayers," Biophysical Journal, vol. 97, no. 1, pp. 40-49, 2009.

[13] H. D. Herce and A. E. Garcia, "Molecular dynamics simulations suggest a mechanism for translocation of the HIV-1 TAT peptide across lipid membranes," Proceedings of the National Academy of Sciences of the United States of America, vol. 104, no. 52, pp. 20805-20810, 2007.

[14] F. Madani, S. Lindberg, Ü. Langel, S. Futaki, and A. Graeslund, "Mechanisms of cellular uptake of cell-penetrating peptides," Journal of Biophysics, vol. 2011, Article ID 414729, 10 pages, 2011.

[15] M. C. Morris, S. Deshayes, F. Heitz, and G. Divita, "Cellpenetrating peptides: from molecular mechanisms to therapeutics," Biology of the Cell, vol. 100, no. 4, pp. 201-217, 2008.

[16] E. Eiríksdóttir, K. Konate, Ü. Langel, G. Divita, and S. Deshayes, "Secondary structure of cell-penetrating peptides controls membrane interaction and insertion," Biochimica et Biophysica Acta, vol. 1798, no. 6, pp. 1119-1128, 2010.

[17] L. Liu, Y. Fang, Q. Huang, and J. Wu, "A rigidity-enhanced antimicrobial activity: a case for linear cationic $\alpha$-helical peptide HP(2-20) and its four analogues," PLoS One, vol. 6, no. 1, Article ID e16441, 2011. 
[18] A. Elmquist, M. Hansen, and Ü. Langel, "Structure-activity relationship study of the cell-penetrating peptide pVEC," Biochimica et Biophysica Acta, vol. 1758, no. 6, pp. 721-729, 2006.

[19] S. S. Izrailev, S. Isralewitz B, D. Kosztin et al., "Steered molecular dynamics," in Computational Molecular Dynamics: Challenges, Methods, Ideas, P. H. Deuflhard, B. Leimkuhler, A. E. Mark, S. Reich, and R. D. Skeel, Eds., pp. 39-65, Springer, Berlin, Germany, 1998.

[20] A. C. Lorenzo and P. M. Bisch, "Analyzing different parameters of steered molecular dynamics for small membrane interacting molecules," Journal of Molecular Graphics and Modelling, vol. 24, no. 1, pp. 59-71, 2005.

[21] H. Huang, E. Ozkirimli, and C. B. Post, "Comparison of three perturbation molecular dynamics methods for modeling conformational transitions," Journal of Chemical Theory and Computation, vol. 5, no. 5, pp. 1304-1314, 2009.

[22] S. Izrailev, S. Stepaniants, and K. Schulten, "Applications of steered molecular dynamics to protein-ligand/membrane binding," Biophysical Journal, vol. 74, no. 2, pp. A177-A177, 1998.

[23] M. Ø. Jensen, Y. Yin, E. Tajkhorshid, and K. Schulten, "Sugar transport across lactose permease probed by steered molecular dynamics," Biophysical Journal, vol. 93, no. 1, pp. 92-102, 2007.

[24] H. Lu, B. Isralewitz, A. Krammer, V. Vogel, and K. Schulten, "Unfolding of titin immunoglobulin domains by steered molecular dynamics simulation," Biophysical Journal, vol. 75, no. 2, pp. 662-671, 1998.

[25] O. V. Levtsova, M. Y. Antonov, D. Y. Mordvintsev, Y. N. Utkin, K. V. Shaitan, and M. P. Kirpichnikov, "Steered molecular dynamics simulations of cobra cytotoxin interaction with zwitterionic lipid bilayer: no penetration of loop tips into membranes," Computational Biology and Chemistry, vol. 33, no. 1, pp. 29-32, 2009.

[26] A. Babakhani, A. A. Gorfe, J. Gullingsrud, J. E. Kim, and J. A. McCammon, "Peptide insertion, positioning, and stabilization in a membrane: insight from an all-atom molecular dynamics simulation," Biopolymers, vol. 85, no. 5-6, pp. 490-497, 2007.

[27] C.-W. Tsai, N. Y. Hsu, C. H. Wang et al., "Coupling molecular dynamics simulations with experiments for the rational design of indolicidin-analogous antimicrobial peptides," Journal of Molecular Biology, vol. 392, no. 3, pp. 837-854, 2009.

[28] W. Humphrey, A. Dalke, and K. Schulten, "VMD: visual molecular dynamics," Journal of Molecular Graphics, vol. 14, no. 1, pp. 33-38, 1996.

[29] A. Elmquist, M. Hansen, and Ü. Langel, "Structure-activity relationship study of the cell-penetrating peptide pVEC," Biochimica et Biophysica Acta, vol. 1758, no. 6, pp. 721-729, 2006.

[30] P. Joanne, C. Galanth, N. Goasdoué et al., "Lipid reorganization induced by membrane-active peptides probed using differential scanning calorimetry," Biochimica et Biophysica Acta, vol. 1788, no. 9, pp. 1772-1781, 2009.

[31] J. C. Phillips, R. Braun, W. Wang et al., "Scalable molecular dynamics with NAMD," Journal of Computational Chemistry, vol. 26, no. 16, pp. 1781-1802, 2005.

[32] A. D. MacKerell, D. Bashford, M. Bellott et al., "All-atom empirical potential for molecular modeling and dynamics studies of proteins," Journal of Physical Chemistry B, vol. 102, no. 18, pp. 3586-3616, 1998.

[33] S. E. Feller and A. D. MacKerell, "An improved empirical potential energy function for molecular simulations of phospholipids," Journal of Physical Chemistry B, vol. 104, no. 31, pp. 7510-7515, 2000.
[34] W. L. Jorgensen, "Transferable intermolecular potential functions for water, alcohols, and ethers. Application to liquid water," Journal of the American Chemical Society, vol. 103, no. 2, pp. 335340, 1981.

[35] T. Darden, D. York, and L. Pedersen, "Particle mesh Ewald: an $\mathrm{N} \cdot \log (\mathrm{N})$ method for Ewald sums in large systems," The Journal of Chemical Physics, vol. 98, no. 12, pp. 10089-10092, 1993.

[36] G. J. Martyna, D. J. Tobias, and M. L. Klein, "Constant pressure molecular dynamics algorithms," The Journal of Chemical Physics, vol. 101, no. 5, pp. 4177-4189, 1994.

[37] S. E. Feller, Y. Zhang, R. W. Pastor, and B. R. Brooks, "Constant pressure molecular dynamics simulation: the Langevin piston method," The Journal of Chemical Physics, vol. 103, no. 11, pp. 4613-4621, 1995.

[38] I. Vorobyov, L. Li, and T. W. Allen, "Assessing atomistic and coarse-grained force fields for protein-lipid interactions: the formidable challenge of an ionizable side chain in a membrane," Journal of Physical Chemistry B, vol. 112, no. 32, pp. 9588-9602, 2008.

[39] H. D. Herce, A. E. Garcia, J. Litt et al., "Arginine-rich peptides destabilize the plasma membrane, consistent with a pore formation translocation mechanism of cell-penetrating peptides," Biophysical Journal, vol. 97, no. 7, pp. 1917-1925, 2009.

[40] M. J. Harms, J. L. Schlessman, G. R. Sue, and B. Garci'a-Moreno, "Arginine residues at internal positions in a protein are always charged," Proceedings of the National Academy of Sciences of the United States of America, vol. 108, no. 47, pp. 18954-18959, 2011.

[41] J. L. MacCallum, W. F. D. Bennett, and D. P. Tieleman, "Partitioning of amino acid side chains into lipid bilayers: results from computer simulations and comparison to experiment," Journal of General Physiology, vol. 129, no. 5, pp. 371-377, 2007.

[42] J. L. MacCallum, W. F. D. Bennett, and D. P. Tieleman, "Distribution of amino acids in a lipid bilayer from computer simulations," Biophysical Journal, vol. 94, no. 9, pp. 3393-3404, 2008.

[43] J. L. MacCallum, W. F. Bennett, and D. P. Tieleman, "Transfer of arginine into lipid bilayers is nonadditive," Biophysical Journal, vol. 101, no. 1, pp. 110-117, 2011.

[44] C. W. Tsai, N. Y. Hsu, C. H. Wang et al., "Coupling molecular dynamics simulations with experiments for the rational design of indolicidin-analogous antimicrobial peptides," Journal of Molecular Biology, vol. 392, no. 3, pp. 837-854, 2009.

[45] Z. Bryant, V. S. Pande, and D. S. Rokhsar, "Mechanical unfolding of a $\beta$-hairpin using molecular dynamics," Biophysical Journal, vol. 78, no. 2, pp. 584-589, 2000.

[46] C. Jarzynski, "Nonequilibrium equality for free energy differences," Physical Review Letters, vol. 78, no. 14, pp. 2690-2693, 1997.

[47] J. Liphardt, S. Dumont, S. B. Smith, I. Tinoco, and C. Bustamante, "Equilibrium information from nonequilibrium measurements in an experimental test of Jarzynski's equality," Science, vol. 296, no. 5574, pp. 1832-1835, 2002.

[48] S. Park and K. Schulten, "Calculating potentials of mean force from steered molecular dynamics simulations," Journal of Chemical Physics, vol. 120, no. 13, pp. 5946-5961, 2004. 

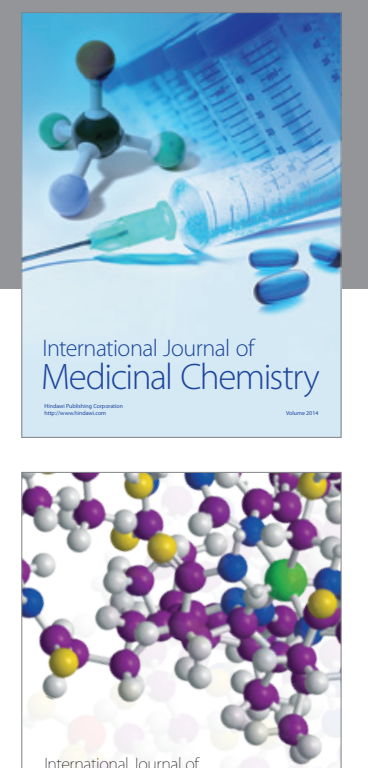

\section{Carbohydrate} Chemistry

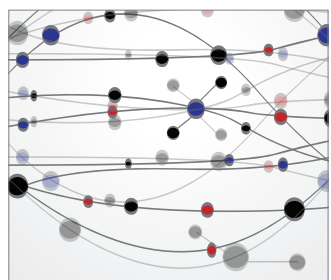

The Scientific World Journal
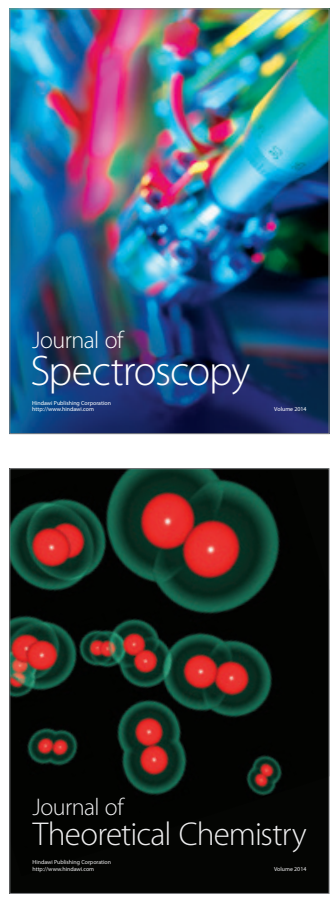
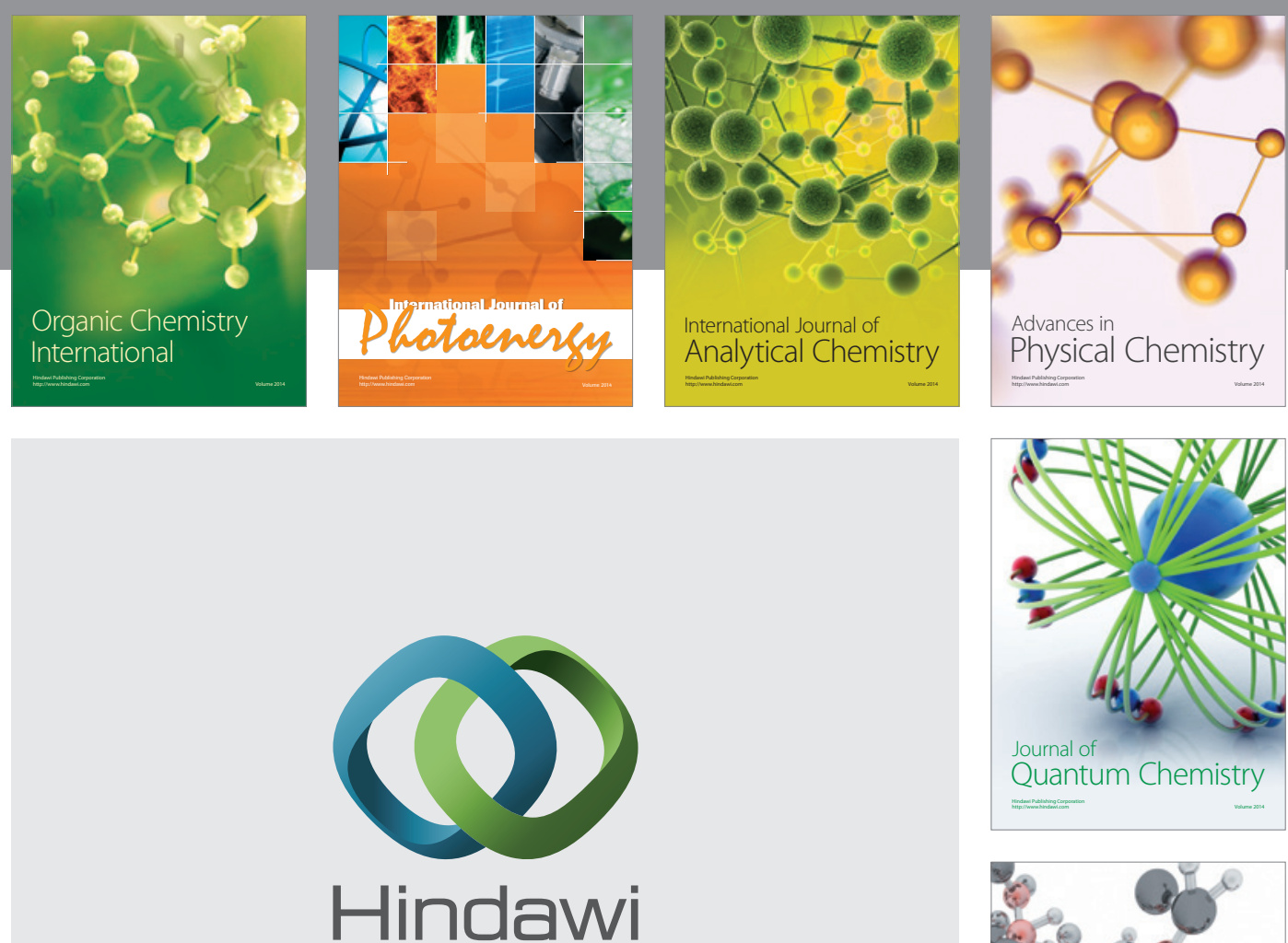

Submit your manuscripts at

http://www.hindawi.com

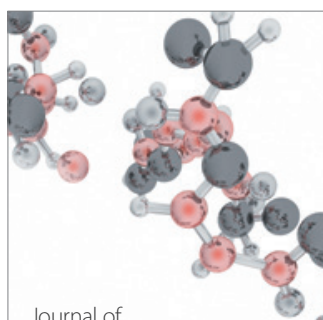

Analytical Methods

in Chemistry

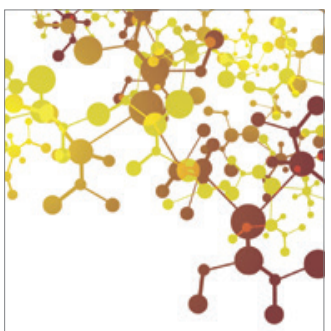

Journal of

Applied Chemistry

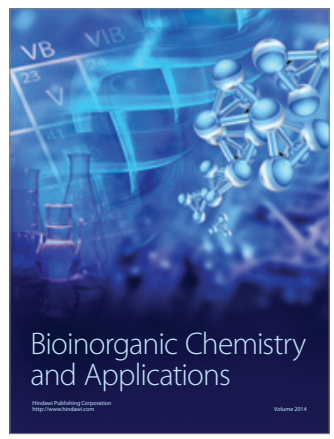

Inorganic Chemistry
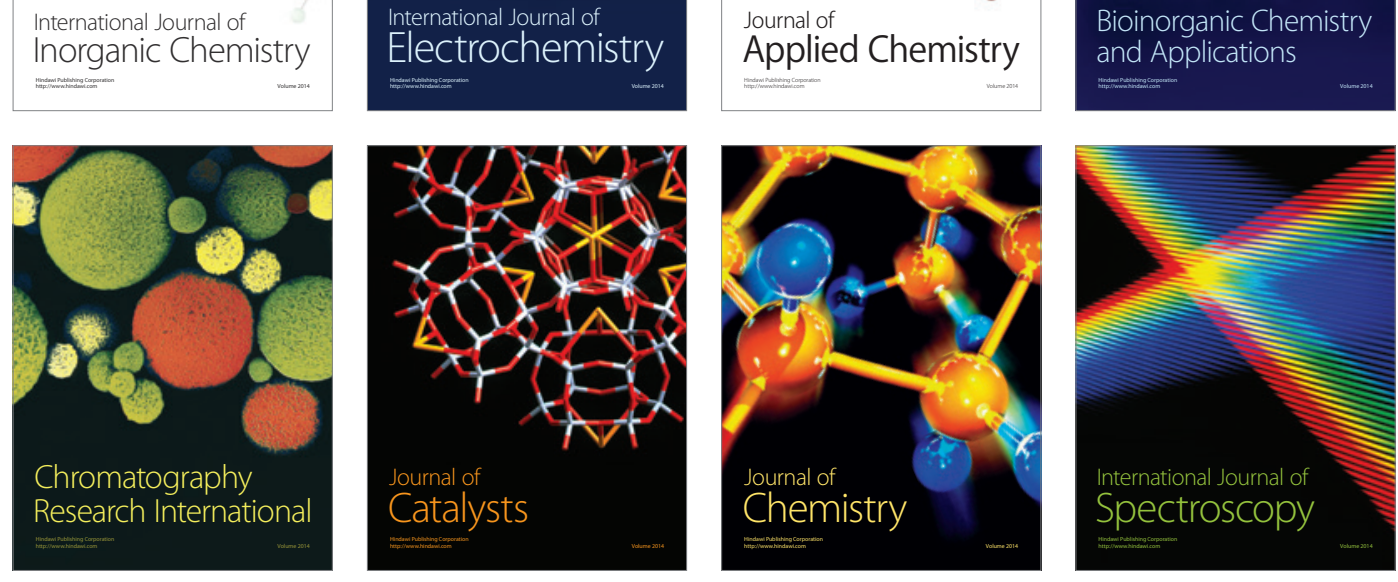